\title{
A Safety Evaluation Model of Intersections under Mixed Traffic Conditions Using Traffic Conflicts and Cloud Model
}

\author{
Xuan Can Vuong $\mathbb{D D}^{1,2}$ Rui-Fang Mou, ${ }^{1}$ Trong Thuat Vu, ${ }^{2}$ Van Hung Tran, \\ and Chi Trung Nguyen ${ }^{2}$ \\ ${ }^{1}$ School of Transportation and Logistics, Southwest Jiaotong University, Chengdu 611756, China \\ ${ }^{2}$ University of Transport and Communications, Hanoi 100000, Vietnam \\ Correspondence should be addressed to Xuan Can Vuong; vuongcan@utc.edu.vn
}

Received 17 July 2021; Revised 16 October 2021; Accepted 10 November 2021; Published 23 November 2021

Academic Editor: Sara Moridpour

Copyright (c) 2021 Xuan Can Vuong et al. This is an open access article distributed under the Creative Commons Attribution License, which permits unrestricted use, distribution, and reproduction in any medium, provided the original work is properly cited.

\begin{abstract}
Safety evaluation of traffic conflict is a very important and challenging issue in evaluating intersection safety under incomplete traffic accident data conditions and is also one of the main safety surrogate measures of analyzing accident data recently. It helps to analyze and solve intersection problems comprehensively and deeply. From there, it helps to improve traffic safety as well as reduce the risk of traffic accidents at intersections. Various evaluation methods based on traffic conflict have been proposed to make conflict safety levels at intersections more consistent and objective. However, a major concern is that many existing measurements are still subjective and are not easy to obtain uniformly. This study aimed to develop a model for safety evaluation at intersections in a comprehensive way that may be expected to directly link to the severity of the accident from different evaluation indicators. First, the three factors, including time to collision (TTC), conflicting speed (CS), and deceleration rate (DR) to avoid a crash, are introduced into safety evaluation of conflicts as the indicators. And then, as regards the fuzziness and randomness of the evaluation indicators, the qualitative concept has to be converted into a quantitative one utilizing cloud model, which implements the natural transformation between the qualitative concept of the safety level of traffic conflict and the membership degree of the evaluation indicators corresponding to the different safety levels. Finally, an indicator weight model is built based on the information entropy and the AHP method to determine the safety level. We illustrate the practical implementation of the proposed method using actual data of a typical signalized intersection from Hanoi City of Vietnam. The results indicate that traffic conflict analyzed by the proposed method was appropriate with actual state of the intersection, and the proposed method is simple, effective, and feasible, so it has a certain application value.
\end{abstract}

\section{Introduction}

The probability that a severe and/or fatal accident occurs at an intersection is higher than elsewhere, so the intersection safety is a crucial component of traffic safety. According to the statistics of accidents, traffic accidents at intersections account for a range of $10 \%$ to $40 \%$ of the total accidents in the world every year. For example, the fatalities at intersections accounted for around $20 \%$ of the total fatalities in the EU in the period 2001-2010 [1]; the road accidents accounted for about $40 \%$ of the total accidents in the United States in 2008 [2] and around 30\% in China [3]. There were around $10 \%$ [4] and $45 \%$ [5] of the total accidents happened at intersections in Hanoi City and Ho Chi Minh City, respectively. Hence, the safety situation of urban intersections has an important effect on traffic safety of the whole road network. It is necessary to do more research studies about the safety prediction and evaluation for urban intersections. The traditional methods (e.g., random-effects models, generalized estimating equations, and Markov chain Monte Carlo [6]) are commonly used, and they fit in several situations well. They can express intersection safety directly, but in some cases, such as limited time periods, historical data availability, and reporting errors, the analysis of accident data based on these methods has many drawbacks. Especially in low- 
and middle-income countries such as Vietnam, obtaining reliable accident data is a difficult task due to the poor quality of historical accident data [7]. As a nonaccident safety evaluation method, traffic conflict is introduced to evaluate the safety of intersections recently as one of the surrogate safety measures of analyzing crash data [8]. It is "an observable situation in which two or more road users approach each other in space and time to such an extent that there is a risk of collision if their movements remained unchanged" [9]. Traffic conflicts not only occur more frequently than crashes but also can be observed or simulated simply and frequently.

The main indicators used in the safety evaluation using traffic conflicts are time to collision (TTC), postencroachment time (PET), deceleration rate (DR) to avoid a crash, and gap time (GT). Of these, the TTC is commonly used, and lower its value indicates a higher likelihood of accidents, but cannot be directly linked to the severity of the accident [10]. Thus, it is more intuitive and reasonable to use TTC with some parameters such as speed and deceleration rate to evaluate the safety. Hyden [11] first combined the TTC and the conflicting speed (CS) to establish a TTC-CS relationship diagram, and according to the diagram, traffic conflicts were classified into two categories: nonserious conflicts and serious conflicts. Li et al. [12] used fuzzy control to identify the severity of rear-end conflicts on the basis of Hyden's method, and the severity was classified into three categories, including serious, slight, and normal. Similarly, $\mathrm{Hu}$ [13] introduced the deceleration rate, the CS, and the TTC as evaluation indicators and used a fuzzy comprehensive method to determine the conflict severity levels, which consist of safety, general conflict, moderate conflict, and serious conflict. Hu et al. [14] introduced the time difference to collision and the vehicle speed as evaluation indicators and established the conflict severity model based on fuzzy control. Ren et al. [15] used the self-organizing map feature neural network method using the TTC, the PET, and the deceleration to safety time (DST) to evaluate conflict severity between pedestrian and vehicle. Regarding quantitative models to evaluate safety conditions in an intersection using indicators of traffic conflicts, the models mainly used the number of conflicts (e.g., grey clustering model [16] and fuzzy clustering model [17]). Some other parameters of traffic conflicts can also be used to indicate safety conditions of an intersection, such as conflict index of conflict angle [6] and intersection conflict index using the level of conflict risk $[18,19]$. These models make a useful contribution to safety evaluation. Most of them are based on fuzzy logic methods or neural network methods to combine multiple indicators, but these methods have some defects in dealing with the actual uncertainty. For example, fuzzy logic (or grey system) is not accurate enough in the concept of membership function and cannot express the randomness of the subjective judgment of the decision-making; the neural network method is difficult to find a learning algorithm and determine the difference coefficient $[20,21]$. Besides, it is known that the conflict severity is rarely reported considering the possibility of accident and the consequences of accident simultaneously.

There are many factors affecting the severity of traffic conflicts, and it is difficult to accurately quantify the model. Moreover, the traffic conflict is a complex process, the conflict situation is different, and the severity of the conflict is different. The safety evaluation based on conflict severity requires a comprehensive consideration of multiple indicators, which are the random and fuzzy attributes with their critical values; i.e., the conflict severity is also uncertain. Hence, there are two types of uncertainty that should be considered in the safety evaluation using conflict severity: (1) randomness, which is often exhibited in the generated event of a traffic conflict; and (2) fuzziness, which is often reflected in the classification standard. For engineering uncertainty, Li et al. [22] proposed the concept of a cloud model, which can realize the natural transformation between qualitative concepts and quantitative descriptions. So far, the cloud model has been widely applied in many different fields [23], such as multicriteria group decision-making [24], data mining [25], and intelligent control [26]. However, a little research studies on the use of cloud models to evaluate traffic safety have been reported at home and abroad.

From the above point of view, this study uses the three basic factors, including TTC, CS, and DR as evaluation indicators, where the TTC represents the possibility of traffic accidents and the CS and DR represent the severity of traffic accident. The safety evaluation model is proposed using a cloud model and traffic conflicts to evaluate the intersections in the context of Vietnamese transport where mixed traffic flow with the interaction and conflicts between motorcycles themselves and different types of vehicles becomes very complicated. It is also one of the main causes of traffic safety problems in urban areas in Vietnam [27]. Hence, the evaluation of traffic safety based on the reasonable model will be an important basis for proposing countermeasures to improve traffic safety. In this study, we should focus on the determination of the location of serious traffic conflicts (i.e., lower safety level), and through the traffic conflict heat map, it can be more intuitive to reflect the serious position of conflicts in the intersection and then the corresponding improvement measures were taken. Tracker software [28] is used to get the number and indicators of traffic conflicts in case study of the selected intersection. And then, we make a quantitative evaluation of intersection safety and demonstrate the distribution of conflicts in the intersection visually in the form of the distribution maps.

\section{Research Model}

2.1. Cloud Model Theory. The cloud model, which was proposed by professor Deyi $[22,29]$ based on the traditional fuzzy set theory and the statistical probability theory, was a new cognition model for uncertainty. It can implement the uncertainty transformation between a qualitative concept and its quantitative value of the 
numeral. Let $U$ be the set $U=\{x\}$, and as a quantitative domain represents by an exact value, $T$ is the qualitative concept of $U$. If quantitative value $x$ is a random realization of concept $T$, and $x \in U$, therefore, $\mu(x)$, which refers to the membership degree of $x$ in $T$, is a random number with a stable tendency.

$$
\mu(x): U \longrightarrow[0,1], \quad \forall x \in U, x \longrightarrow \mu(x) .
$$

The distribution of $x$ in $U$ is called the cloud model, or referred to as cloud, and $(x, \mu)$ is called cloud drop. The digital features of the cloud model to reflect the overall characteristics of the concept are represented by three digital values: expected value $(E x)$, entropy $(E n)$, and hyper-entropy $(\mathrm{He})$. Of these, the expected value is described as the center of the whole cloud drops, reflecting the digital domain coordinates, which have the most representative of the concept. Entropy is the measurement of the qualitative concept of randomness, reflecting the representing qualitative concept of the dispersion degree of the cloud drops. Hyperentropy is the dispersion degree of the entropy En, which reflects the cloud thickness. If the membership degree $\mu(x)$ of $x$ in $T$ satisfies the following equation:

$$
\mu(x)=\exp \left[\frac{-(x-E x)^{2}}{2 E^{\prime} n^{2}}\right],
$$

where $x \sim N\left(E x, E^{\prime} n^{2}\right), E^{\prime} n \sim N\left(E n, H e^{2}\right)$, and then, the distribution of $x$ in $U$ is called normal cloud, which is generated by the forward cloud generator (FCG). The FCG is described as the algorithm to generate a quantity of cloud drops of the normal cloud model using the three digital features, which are shown in Figure 1. The normal FCG achieves the acquisition of the range of quantitative data and the distribution laws from the qualitative information expressed by language value. It is a common and important tool to represent a language concept.

In addition to the normal cloud, half-down cloud, halfup cloud, and trapezoidal cloud are used. The specific concept can be referred to as Li et al. [30]. The normal cloud by the FCG is presented in the following [23]:

(i) Step 1: according to the three digital characteristics of cloud, a normally distributed random number $E^{\prime} n_{i}$ with expectation $E n$ and variance $H e^{2}$ is generated.

(ii) Step 2: a normally distributed random number $x_{i}$ with expectation $E x$ and variance $E^{\prime} n_{i}^{2}$ is generated.

(iii) Step 3: according to Step 1 and Step 2, $\mu_{i}=\exp \left[-(x-E x)^{2} / 2 E^{\prime} n_{i}^{2}\right]$ is calculated, and it is the certainty degree of $x_{i}$ belonging to qualitative concept $T$.

(iv) Step 4: $\left(x_{i}, \mu_{i}\right)$ is a cloud droplet on the domain, and Steps 1-3 are repeated until $N$ number of cloud droplets generated so far. For example, Figure 2 depicts a cloud model of three digital features.

From the " $3 E n$ rule" of normal clouds, the digital features $E x, E n$, and $H e$ can be computed by the threshold values of each parameter as follows:

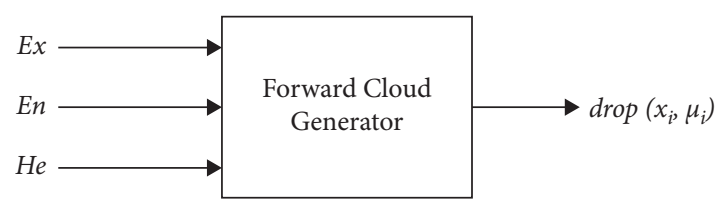

Figure 1: Forward cloud generator.

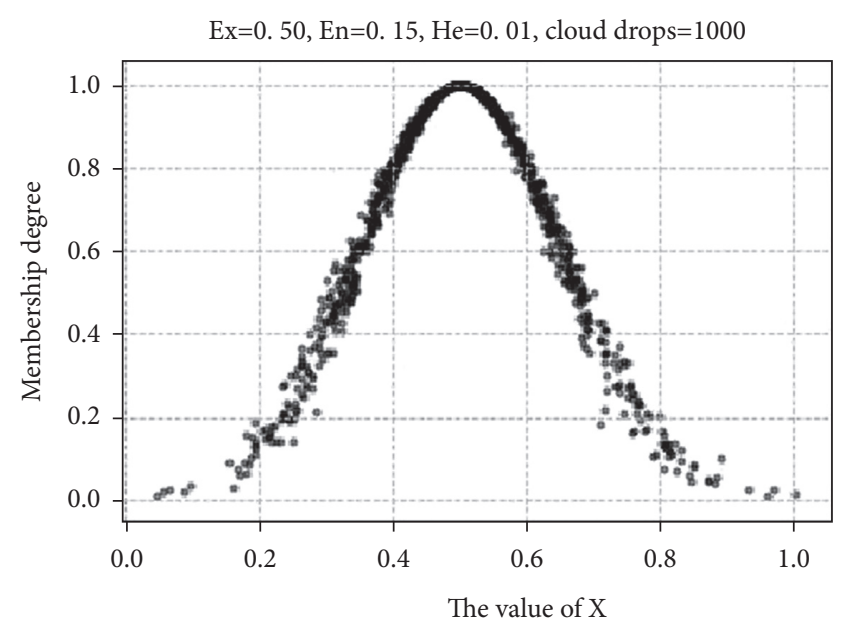

Figure 2: Digital features of normal cloud.

$$
\left\{\begin{array}{l}
E x=\frac{\left(X_{\max }+X_{\min }\right)}{2}, \\
E n=\frac{\left(X_{\max }-X_{\min }\right)}{6}, \\
H e=k,
\end{array}\right.
$$

where $X_{\min }$ and $X_{\max }$ are the minimum and maximum values of concept, respectively; $k$ is a constant and can be adjusted according to the fuzzy threshold of the variable. For single-boundary variables, such as $\left(X_{\min },+\infty\right]$ and $\left[-\infty, X_{\max }\right)$, these expected values can be determined based on the maximum or minimum value. According to the principle of the FCG, a specific point in the domain $U$ is given, and through the FCG, certainty degree distribution belonging to this concept is produced; this is called antecedent cloud generator, also called $X$ condition cloud generator. For any sample, $x_{i j}^{*}$ is evaluated, and the membership degree of the $j$ - th attribute of the $i$-th object belonging to the $k$-th level can be calculated.

$$
\mu_{i k}\left(x_{i j}^{*}\right)=\exp \left[\frac{-\left(x_{i j}^{*}-E x_{i j}\right)^{2}}{2\left(E n_{i j}^{\prime}\right)^{2}}\right] .
$$

According to the principle of $X$-condition cloud generator, the mean of the membership degree of $x_{i j}^{*}$ can be obtained and used as the final membership degree, as shown below. 


$$
\overline{\mu_{i j}}\left(x_{i j}^{*}\right)=\sum_{h=1}^{H_{0}} \mu_{i j}\left(x_{i j}^{*}\right),
$$

where $H_{0}$ is the number of cloud droplets through $X$ condition cloud generator $\left(H_{0}=100\right)$.

\subsection{Safety Evaluation of Intersections Using Cloud Model and} Traffic Conflicts. Safety evaluation of intersections using cloud model and traffic conflicts can be summarized as follows:

(i) Step 1: construction of indicator system using indicator of the traffic conflicts at intersections

(ii) Step 2: discretization of continuous attributes, i.e., determination of criteria of indicators

(iii) Step 3: determination of parameters $(E x, E n, H e)$ of each indicator, based on given levels, and the corresponding bilateral boundaries, before the cloud is completely modeled

(iv) Step 4: calculation of the indicator weights

(v) Step 5: substitution of the observed data into cloud models repeatedly to obtain the distributions of certainty degrees, and further final outcomes corresponding to all levels

(vi) Step 6: determination of safety level based on the level with correlation coefficient calculated from the cloud model and the indicator weight model

(vii) Finally, determination of safety index of the intersection

2.2.1. Construction of Cloud Model for Indicator System. The indicator system of safety evaluation at intersection should be constructed based on some parameters of traffic conflict, including TTC, CS, and DR. TTC is "the expected time for two road users to collide if they remain at their present speed, direction, and on the same trajectory" [31]. The higher TTC values indicate the lower severity. CS is the speed of the road user during evasive action and is used to measure the TTC. DR is the required deceleration rate to avoid a collision if the conflicting target vehicle maintains its speed and trajectory. The higher CS and DR values indicate the higher severity of the traffic conflict that occurs. The evaluation indicators can be obtained via one or several methods such as field observation, video analysis, and traffic simulations. In this study, these indicators are collected by video analysis.

\subsubsection{Discretization of Continuous Attributes. Let} $U=\left\{u_{1}, u_{2}, u_{3}, \ldots, u_{n}\right\}$ be the evaluation objects whose evaluation level is a qualitative concept, which can be divided into $p$ levels with the great uncertainties in evaluation process; $m$ attributes (evaluation indicators) need to be considered, i.e., $C=\left\{c_{1}, c_{2}, c_{3}, \ldots, c_{m}\right\}$. Hence, each conflict point of the domain is characterized by $m$ evaluation indicators, that is, $u_{i}=\left(x_{i 1}, x_{i 2}, \ldots, x_{i m}\right)$; let $V=\left\{v_{1}, v_{2}, v_{3}, \ldots, v_{p}\right\}$ is the set of evaluation level; $i=1,2, \ldots, n ; j=1,2, \ldots, m$; and $k=1,2, \ldots, p$. In this study, the evaluation objects are traffic conflict points at intersections. The three factors, including TTC, CS, and DR, are used as evaluation indicators, i.e., $C=\{T T C, C S, D R\}$ and $m=3$.

Currently, the safety levels are not uniform, usually from 3 to 5 levels. Some scholars used 4 levels to divide safety levels of the intersection; for example, Yu and Wang [16] used grey clustering method to divide safety levels into 4 levels, corresponding to excellent, good, medium, and poor; Li et al. [32] also applied grey system to divide the rank safety performance of signalized intersections into 4 levels, including excellent, good, medium, and poor; and Huang et al. [17] used the ratio of traffic conflict and mixed passenger car unit through fuzzy clustering method to divide safety levels into 4 clusters. On that basis, in this study, the safety levels of each indicator, as well as the safety level of traffic conflict points, are divided into four levels, namely, safety (level I), relative safety (level II), less safety (level III), and danger (level IV), i.e., $V=\{I, I I, I I I, I V\}$ and $p=4$.

In reviewing previous studies, it can be seen that the thresholds of the indicator are inconsistent. Generally, the TTC $[18,33,34]$ in seconds is in the range of $[0,3]$, the CS [35-37] in kilometers per hour is in the range of $[0,80]$, and the DR [38] in meters per second squared is in the range of $[0,8]$. Besides, these values are used in many studies to evaluate traffic safety under traffic flow of car dominance, which is very different from heterogeneous traffic like in Hanoi City (Vietnam) where motorcycles are used frequently (over $80 \%$ of trips). Non-lane-based movements of different vehicle types on roads are very complex in comparison with homogeneous traffic like in developed countries [27, 39]. Therefore, directly using the abovementioned threshold values would be difficult to accurately reflect the nature of the mixed traffic flow as Vietnam.

In Vietnam, previous studies showed that most of the road traffic accidents at signalized intersections occur more often during nonpeak hours of traffic flow [5, 40, 41]. Thus, this study just focuses on collecting data at this time to increase practicality. Besides, in the context of mixed traffic flow dominated by motorcycles like in Vietnam, different types of vehicles, which mainly include motorcycles and cars, share the same road space to deal with the lack of lane descriptions. Therefore, the average speed difference in the vehicle types at approaches of an urban intersection is not high (within $3 \mathrm{~km} / \mathrm{h}$ ) [42, 43]. Since then, this study is carried out based on the following assumptions:

(i) Traffic conflict is collected on nonpeak hours of traffic flow when accidents are more likely

(ii) The models in this study do not consider the conflict separately for each type of vehicle; i.e., there is no weight of conflict between motorbikes and other vehicles

(iii) This study also does not consider the conflict of different types of movement separately 
On the basis of the above assumptions, we use cumulative frequency curves of TTC, CS, and DR under a total of 2669 conflict points during nonpeak hours, including 319 conflict points of 2 intersections in Hanoi City [44] and 2350 conflict points of intersections in Ho Chi Minh City, Vietnam [5], using video analysis to determine the thresholds and to discretize the continuous attributes of the indicators according to the safety levels. The thresholds of four levels are selected in accordance with the values of $15 \%$, $40 \%, 60 \%$, and $85 \%$ of the cumulative frequency of each indicator, as the domain of the indicators $[3,6]$. The $\mathrm{cu}-$ mulative frequency curves for indicators are obtained from traffic conflicts observed at 12 intersections as shown in Figures $3-5$. The domain of the indicators is described as shown in Table 1.

\subsubsection{Definitions of Cloud Model for Indicator System.} Based on Table 1 and equation (3), we can set the digital features of the cloud model as shown in Table 2.

From the parameters in Table 2, we can build the cloud model for the evaluation indicators as shown in Figures 6 to 8 .

2.2.4. Determination of the Weight of the Indicator. When evaluating the system, the importance of each indicator may be quite different. Therefore, it is a basic part of an evaluation model to set suitable weights of evaluation indicator to achieve more accuracy and effectiveness. In this study, we use the analytic hierarchy process (AHP) method [45] and the entropy-based method to determine the weight of the indicator, which is calculated as follows:

$$
W_{j}=\frac{w_{A j}+w_{E j}}{\sum_{j=1}^{m}\left(w_{A j}+w_{E j}\right)},
$$

where $W_{A j}$ and $W_{E j}$ are the weights of $j$ th indicator calculated by the AHP method and the entropy-based method, respectively. To assess the score and the weight of each indicator corresponding AHP method, we invite a team of 15 experts with experience in the transport sector in Hanoi City to make a score on a designed questionnaire. To synthesize the assessments of each expert into a single assessment representing the opinions of the whole group, we use the geometric mean method. The entropy-based weight method is commonly used to determine the uncertainty of a system based on the thermodynamic foundation of the modern information theory proposed by Shannon. The information entropy in a system is a disorder measure, which is understood that the greater the information entropy, the higher the degree of disorder and the smaller the utility value of information, and on the other hand, the smaller the information entropy, the lower the degree of disorder and the greater the utility value of information. According to the principle, the entropy weight is calculated as follows.

In the actual project, some indicators are smaller and better, and some indicators are larger and better. The

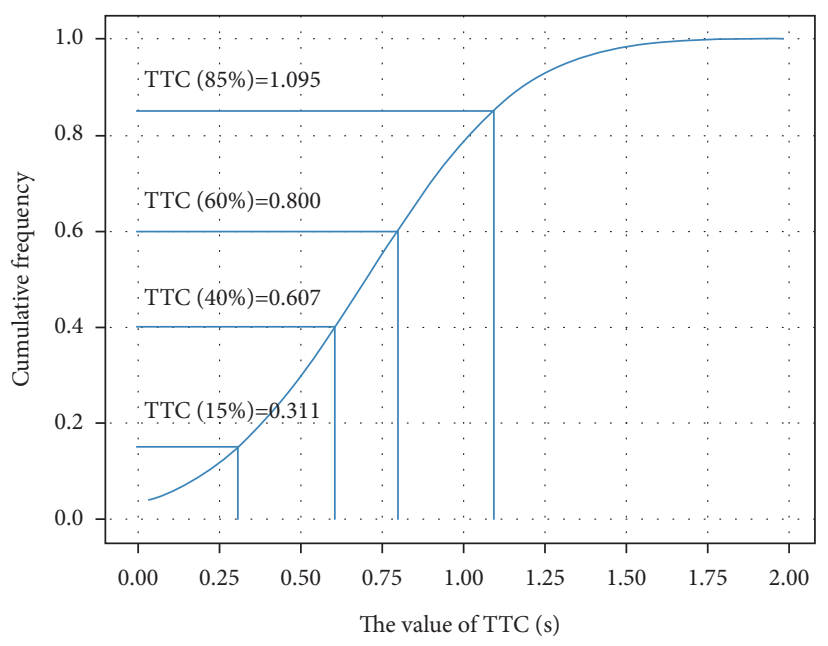

FIgUre 3: Cumulative frequency curve for the TTC.

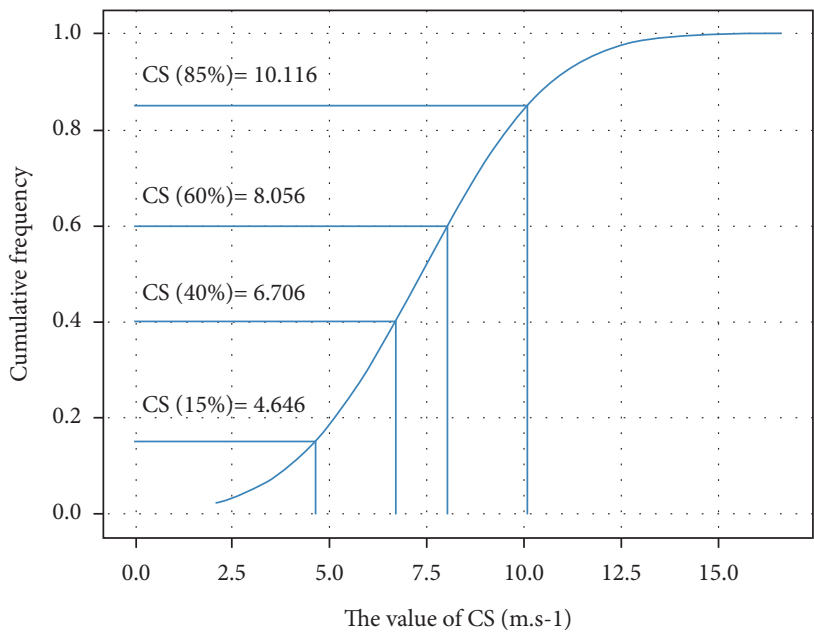

FIgURE 4: Cumulative frequency curve for the CS.

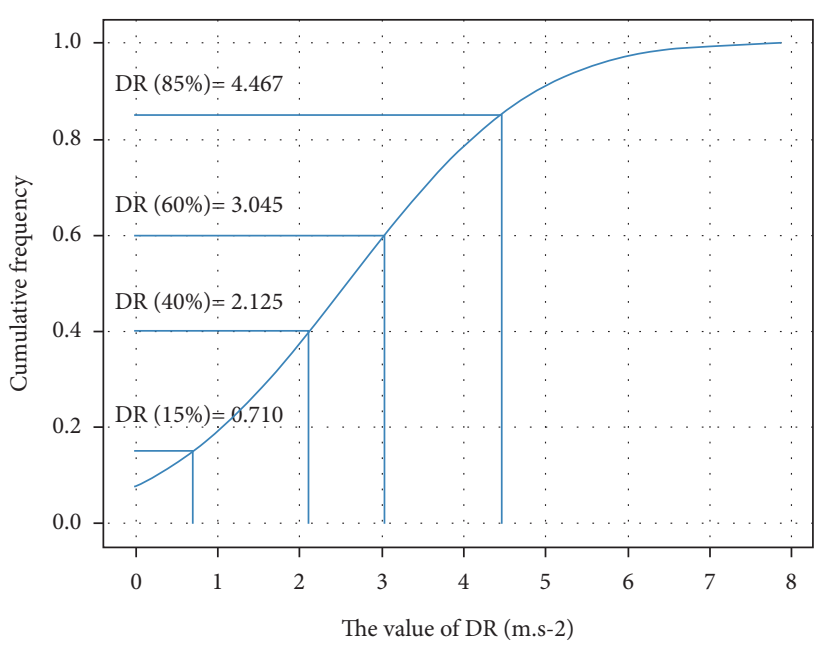

FIgURE 5: Cumulative frequency curve for the DR.

judgment matrix $R_{n m}=\left\{r_{i j}\right\}_{n \times m}$ will be normalized to eliminate the impact of dimension, and the elements of the matrix can be expressed as follows. 
TABLE 1: Domain of the indicators.

\begin{tabular}{lcccc}
\hline Safety level & $(\mathrm{I})$ & $(\mathrm{II})$ & $(\mathrm{III})$ & $(\mathrm{IV})$ \\
\hline TTC $(\mathrm{s})$ & {$[0.800,2]$} & {$[0.607,1.095]$} & {$[0.311,0.800]$} & {$[0,0.607]$} \\
CS $(\mathrm{m} / \mathrm{s})$ & {$[0,6.706]$} & {$[4.646,8.056]$} & {$[6.706,10.116]$} & {$[8.05616]$} \\
DR $\left(\mathrm{m} / \mathrm{s}^{2}\right)$ & {$[0,2.125]$} & {$[0.710,3.045]$} & {$[2.125,4.467]$} & {$[3.045,8]$} \\
\hline
\end{tabular}

Table 2: Digital features $(E x, E n, H e)$ of cloud model of the evaluation indicators.

\begin{tabular}{lccr}
\hline Safety level & TTC & CS & DR \\
\hline (I) & $(1.095,0.098,0.01)$ & $(4.646,0.687,0.05)$ & $(0.710,0.472,0.05)$ \\
(II) & $(0.851,0.081,0.01)$ & $(6.351,0.568,0.05)$ & $(1.878,0.389,0.05)$ \\
(III) & $(0.556,0.082,0.01)$ & $(8.411,0.568,0.05)$ & $(3.296,0.39,0.05)$ \\
(IV) & $(0.311,0.099,0.01)$ & $(10.116,0.687,0.05)$ & $(4.467,0.474,0.05)$ \\
\hline
\end{tabular}

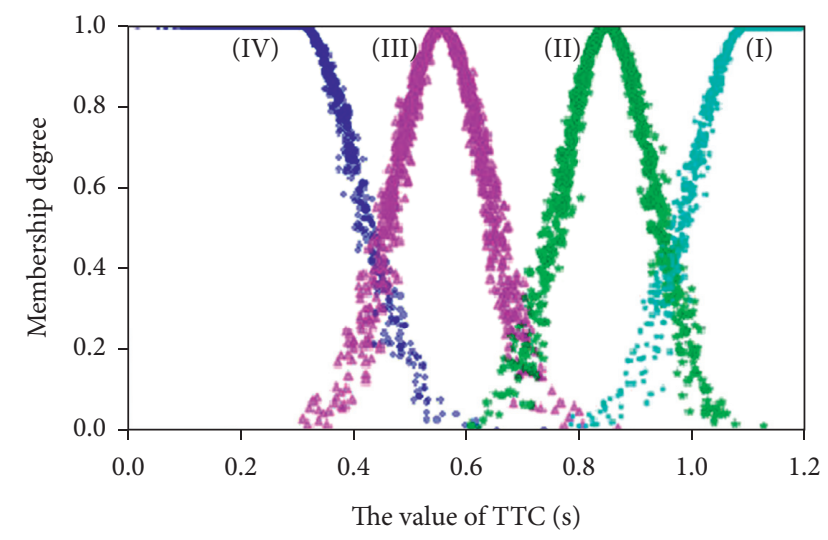

FIgure 6: Cloud model for the TTC.

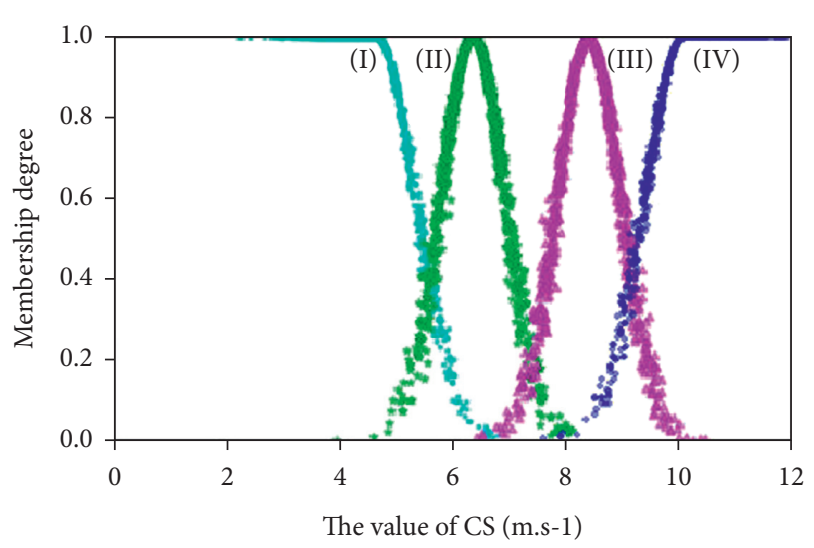

FIgUre 7: Cloud model for the CS.

For smaller and better, such as TTC,

$$
r_{i j}=\frac{\max _{j}\left\{x_{i j}\right\}-x_{i j}}{\max _{j}\left\{x_{i j}\right\}-\min _{j}\left\{x_{i j}\right\}}
$$

For larger and better, such as CS and DR,

$$
r_{i j}=\frac{x_{i j}-\min _{j}\left\{x_{i j}\right\}}{\max _{j}\left\{x_{i j}\right\}-\min _{j}\left\{x_{i j}\right\}}
$$

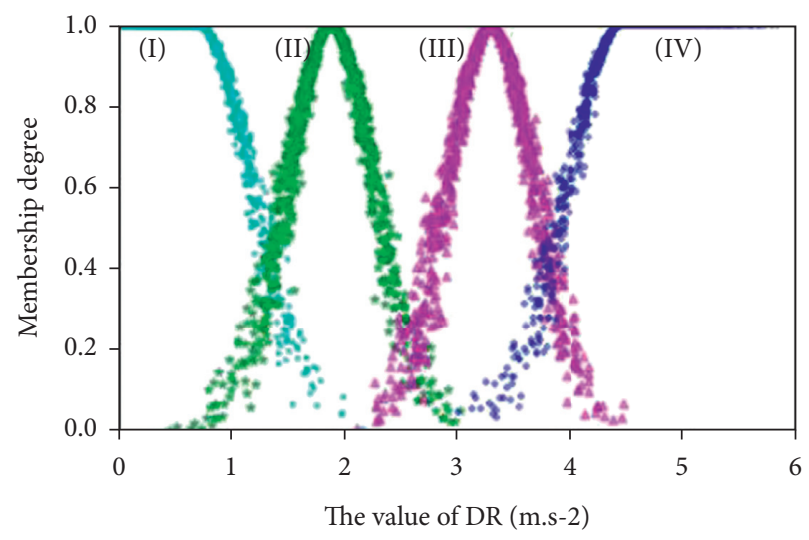

FIgUre 8: Cloud model for the DR.

where $x_{i j}$ is the actual value of the $i$ - th object of the $j-$ th indicator; $\max _{j}\left\{x_{i j}\right\}$ and $\min _{j}\left\{x_{i j}\right\}$ are the maximum and minimum values in all samples of the evaluation indicator, respectively; and $r_{i j}$ is a dimensionless parameter normalized. The normalized information entropy $\left(E_{j}\right)$ of the observed data under the jth indicator can be calculated as follows:

$$
E_{j}=\frac{-1}{\ln (n)} \sum_{i=1}^{n} r_{i j}^{\prime} \ln r_{i j}^{\prime}, r_{i j}^{\prime}=\frac{r_{i j}}{\sum_{i=1}^{n} r_{i j}},
$$

where $E_{j}$ represents the uncertainty of observed data of one criterion with $n$ potential intervals or statements; $r_{i j}^{\prime}$ is the frequency of the $i$ th statement; if $r_{i j}^{\prime}=0$, the entropy calculation will fail. So to avoid this problem, it is necessary to introduce a correction value $(\alpha=1)$ to improve entropy, which is calculated as follows:

$$
r_{i j}^{\prime}=\frac{\alpha+r_{i j}}{\sum_{i=1}^{n}\left(\alpha+r_{i j}\right)} .
$$

The entropy weight of the jth indicator $\left(W_{E j}\right)$ can be attained as follows:

$$
W_{E j}=\frac{1-E_{j}}{\sum_{j=1}^{m}\left(1-E_{j}\right)}, \quad 0<W_{E j} \leq 1, \sum_{j=1}^{m} W_{E j}=1 .
$$


2.2.5. Correlation Coefficient Calculation. The weights of all indicators are determined, and we can compute the normalized correlation coefficient $\sigma_{i k}$ between object $u_{i}$ and $k-$ th level as follows:

$$
\sigma_{i k}=\frac{\sum_{j=1}^{m} w_{j} \mu_{i k}\left(x_{i j}\right)}{\sum_{k=1}^{p} \sum_{j=1}^{m} w_{j} \mu_{i k}\left(x_{i j}\right)},
$$

where $\mu_{i k}\left(x_{i j}\right)$ is the membership degree of the actual value $x_{i j}$ belonging to the $k$ th level. To avoid the decision distortion caused by applying the principle of maximum correlation coefficient and to improve the reliability and stability of the evaluation results, the confidence criterion is used to evaluate the severity level of conflict points. Let $\lambda$ be the confidence level, and the commonly used confidence interval is $0.5 \leq \lambda \leq 0.7$, and this study takes $\lambda=0.6$. If the following equation is satisfied, then the current level is the level $k^{*}$.

$$
k^{*}=\min \left\{k: \sum_{k=1}^{4} \sigma_{i k} \geq \lambda, \quad k=1,2,3,4\right\},
$$

and to rank the conflict points of the same levels, as well as determine correlation coefficient of the level $k^{*}$, this study uses a weighted value $\left(\sigma_{i k *}\right)$, and it is calculated as follows:

$$
\sigma_{i k *}=\frac{\sum_{k=1}^{4} k \sigma_{i k}}{\sum_{k=1}^{4} \sigma_{i k}}
$$

2.2.6. Determination of Safety Index of the Intersection. Safety levels of conflict points exert different influences on the intersection safety conditions. The influence should be embodied by weights. Therefore, safety index of the intersection could be built by weighted summation of relative value of conflict points as shown in the following equation:

$$
\mathrm{SI}=\frac{\sum_{k=1}^{4} W_{G p} \mathrm{TC}_{p}}{\mathrm{MPCU}},
$$

where SI is the safety index of the intersection; $\mathrm{TC}_{P}$ is the hourly conflict of $p$ level; MPCU is the mixed passenger car unit of the hourly entering volumes; and $W_{G p}$ is the weight of $p$ level, which can be determined using order relation analysis (G1 method) $[46,47]$ through the scores provided by 15 experts in this study. The higher the SI value is, the lower the safety conditions are. The SI also can be used to compare and rank the safety conditions of intersections.

\section{Empirical Study}

3.1. Data Source. A typical signalized intersection in Hanoi City, the capital of Vietnam, namely, Ham Nghi-Nguyen Dong Chi intersection (coordinates: longitude $=21.035194$, latitude $=105.763771$ ), is selected for an empirical study. The traffic flow data are gathered using an unmanned aerial vehicle (UAV) video. All traffic movements are captured under dry-weather conditions and during 30 minutes of nonpeak hours. This technique required two corresponding persons in the field to observe proper positions for video recording and mark 4 base points as ground coordinate system, which is used to convert between image coordinate system and real coordinate system when analyzing video on traffic laboratory.

3.2. Data Preprocessing. Video file with resolution of $1920 \times 1080$ and fps of 23 is replayed in a computer and interpreted until entire necessary data are accomplished in the laboratory. The video is reviewed at either slow speed to scan full-required observation of selected intersection or high speed to skip unnecessary data. Also, the recorder allowed the film to stop at any time and any point to a single traffic scene for detailed observation and analysis. In this study, the location and speed of the vehicles regarding time events from the image video file are determined according to image coordinates and then are converted into ground coordinates using Tracker [28], which is a free video analysis and modeling tool built on the Open Source Physics Java framework. The features of object tracking include position, velocity, acceleration overlays and graphs, special effect filters, multiple reference frames, calibration points, line profiles for analysis of spectra and interference patterns, and dynamic particle models. A diagram of calculating indicators using Tracker is shown in Figure 9 [5]. To get the values of indicators, the first step is to find a vehicle that can be potential conflict with other vehicles. And then, according to the speed and location of the vehicles, which are extracted from Tracker software, we can obtain the values of indicators (i.e., TTC, CS, and DR).

A dataset of 126 conflict points at selected intersection in a specific time period is established to evaluate safety levels, as shown in Table 3.

3.3. Determination of Safety Level. According to 2669 conflict points of 12 intersections, the entropy-based weights of the indicators are calculated by equations (7) to (11) as follows: $W_{E 1}=0.234, W_{E 2}=0.284$, and $W_{E 3}=0.482$. Besides, on the basis of expert judgment, the AHP-based weights of the indicators are also determined as follows: $W_{A 1}=0.352, W_{A 2}=0.254$, and $W_{A 3}=0.394$. According to equation (6), finally the weights of the indicators are $W_{1}=0.293, W_{2}=0.269$, and $W_{3}=0.438$. According to the dataset in Table 3, we can easily obtain the correlation coefficients and safety level of conflict points, as shown in Table 4. From the parameters in Table 4, the distribution of conflict points is also reproduced to perform other related analyses, as shown in Figures 10 to 11 .

The 126 conflict points at the intersection after evaluation include 31 points of level I, 44 points of level II, 42 points of level III, and 9 points of level IV, as shown in Figures 10 and 11. From Figure 11, it can be seen that the conflict points are concentrated mainly on left-turn movements dealing with the lack of phase for left-turn vehicles in north-south direction. This is consistent with the actual operation at the intersection. Besides, during the observation period of traffic conflicts at the intersection, the collected entering vehicles are also 5,123 


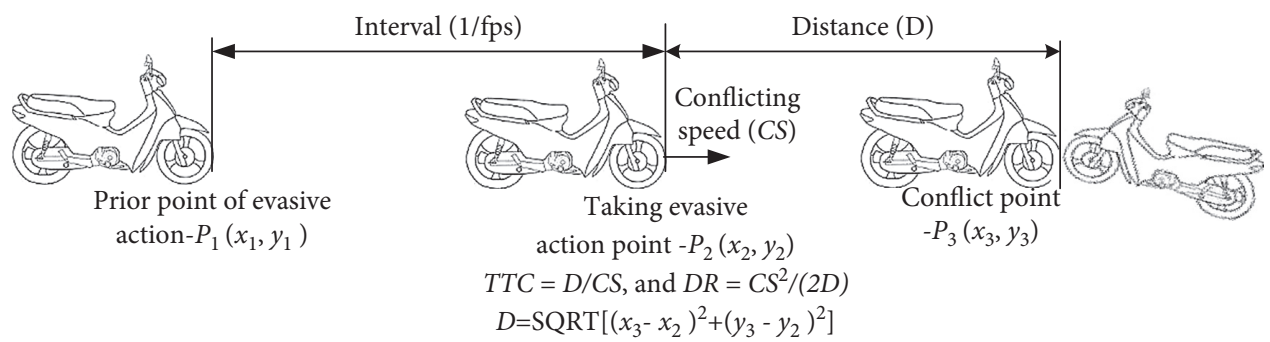

Figure 9: Diagram of calculating indicators.

TABLE 3: The dataset of conflict points.

\begin{tabular}{lccc}
\hline Conflict points & TTC $(\mathrm{s})$ & CS $(\mathrm{m} / \mathrm{s})$ & DR $\left(\mathrm{m} / \mathrm{s}^{2}\right)$ \\
\hline 1 & 0.373 & 2.246 & 3.013 \\
2 & 0.459 & 2.955 & 3.221 \\
3 & 0.638 & 5.079 & 3.983 \\
4 & 0.746 & 3.039 & 2.036 \\
5 & 1.565 & 5.542 & 1.771 \\
6 & 0.779 & 5.521 & 3.545 \\
7 & 0.373 & 3.295 & 4.418 \\
$\ldots$ & $\ldots$ & $\ldots$ & $\ldots$ \\
$\ldots$ & $\ldots$ & $\ldots$ & $\ldots$ \\
126 & 0.597 & 3.096 & 2.591 \\
\hline
\end{tabular}

TABLE 4: Correlation coefficients and safety level of conflict points.

\begin{tabular}{|c|c|c|c|c|c|c|}
\hline Conflict points & (I) & (II) & (III) & (IV) & $\sigma_{* k}$ & Safety level $\left(k^{*}\right)$ \\
\hline 1 & 0.304 & 0.010 & 0.410 & 0.276 & 2.658 & 3 \\
\hline 2 & 0.281 & 0.002 & 0.600 & 0.116 & 2.552 & 3 \\
\hline 3 & 0.281 & 0.043 & 0.347 & 0.329 & 2.724 & 3 \\
\hline 4 & 0.336 & 0.633 & 0.031 & 0.000 & 1.695 & 2 \\
\hline 5 & 0.462 & 0.537 & 0.001 & 0.000 & 1.539 & 2 \\
\hline 6 & 0.145 & 0.341 & 0.435 & 0.079 & 2.448 & 3 \\
\hline 7 & 0.274 & 0.000 & 0.036 & 0.690 & 3.142 & 4 \\
\hline$\cdots$ & $\ldots$ & $\ldots$ & $\ldots$ & $\ldots$ & $\ldots$ & $\cdots$ \\
\hline$\ldots$ & $\ldots$ & $\ldots$ & $\ldots$ & $\ldots$ & $\ldots$ & $\ldots$ \\
\hline 126 & 0.382 & 0.123 & 0.486 & 0.008 & 2.120 & 3 \\
\hline
\end{tabular}

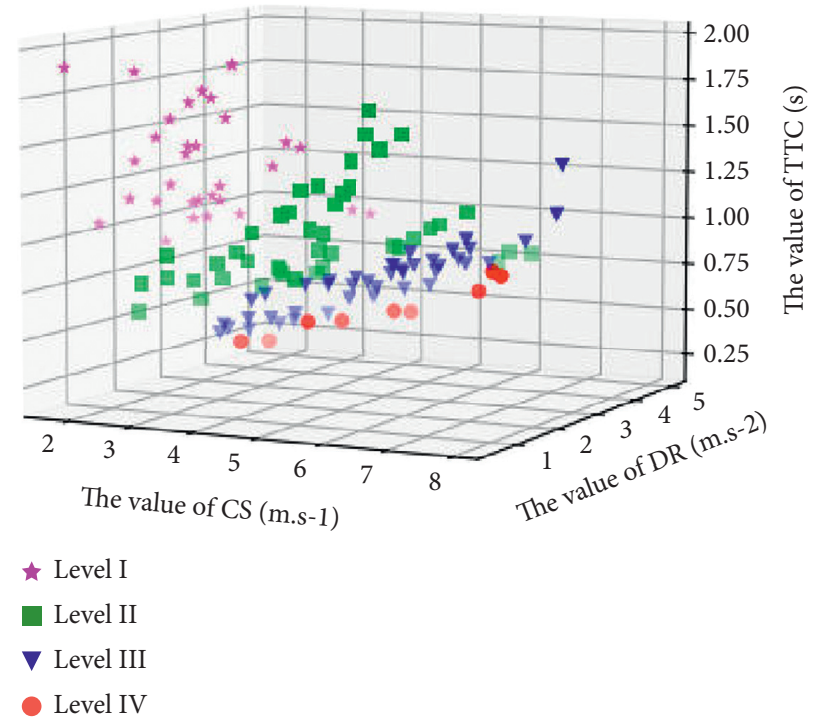

Figure 10: Distribution of safety levels of conflict points at the intersection. 


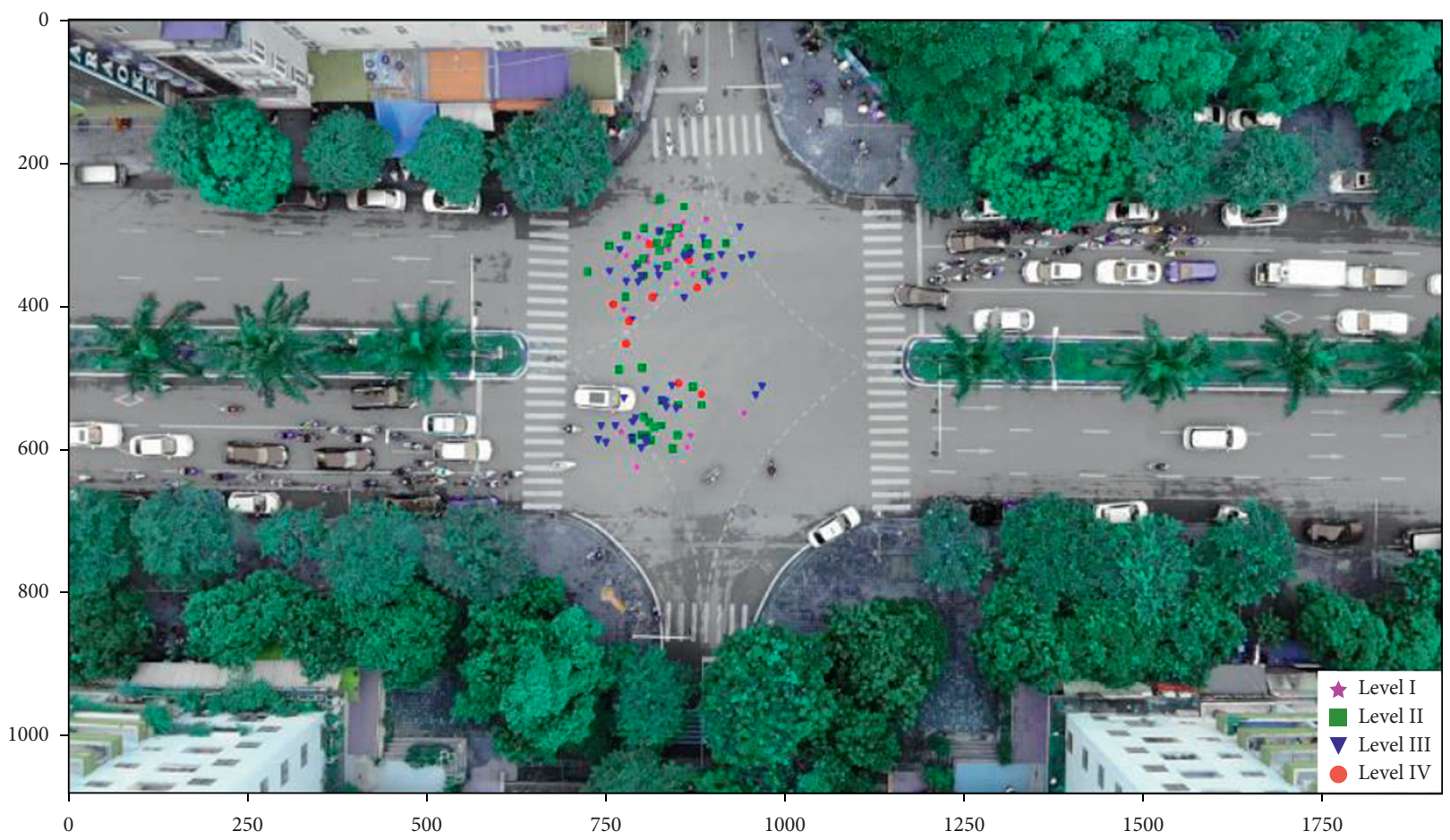

FIgURE 11: Plane of the distribution of conflict points at the intersection.

units, including $703(13.7 \%)$ cars, 4,381 (85.5\%) motorcycles, $16(0.3 \%)$ bicycles, $10(0.2 \%)$ minibuses, $3(0.1 \%)$ buses, and $10(0.2 \%)$ trucks. The number of MPCU converted is 1,416 units based on the PCU factors suggested by Reference [48]. Through the G1 method, we easily determine the weights of the safety levels of the conflict points $\left(w_{G p}\right)$, which are $0.169,0.216,0.273$, and 0.342 corresponding to level I, level II, level III, and level IV, respectively. Based on equation (15), we can easily determine that the safety index of the intersection (SI) is 0.021 . This is the basis for comparison and ranking with other intersections.

\section{Conclusion}

The analysis of traffic conflict is one of the main safety surrogate measures of analyzing accident data. It has the advantages of rapid and quantitative analysis and is widely used at present. In this study, different safety levels of conflict points at intersections were considered in the developing method to evaluate urban intersection safety. The TTC, CS, and DR were taken as evaluation indicators to distinguish conflicts of different safety levels. The method based on cloud model had been developed to calculate the safety levels of conflict points at intersections. The results of the example analysis indicated that the proposed method is intuitive, simple, effective, and feasible. It not only can be useful to estimate the safety condition of intersections but also is the foundation of proposals for safety improvements of intersections.

\section{Data Availability}

The data used to support the findings of this study are available from the corresponding author upon request.

\section{Conflicts of Interest}

The authors declare that they have no conflicts of interest.

\section{Acknowledgments}

This research has been supported by the Ministry of Education and Training of Vietnam, under Grant no. CT2019.05.03.

\section{References}

[1] J. Broughton, P. Thomas, A. Kirk et al., Traffic Safety Basic Facts 2012: Junctions, European Commision, Belgium, Luxembourg, 2013.

[2] E. Choi, Crash Factors in Intersection-Related Crashes: An OnScene Perspective (No. Dot Hs 811 366), US DOT National Highway Traffic Safety Administration, Washinton, DC, USA, 2010.

[3] M. F. Huang and T. L. Chen, "Evaluation of city traffic safety at intersections based on traffic conflict," Journal of Heilongjiang Institute of Technology, vol. 27, no. 1, pp. 10-13, 2013.

[4] M. H. O. A. D. Thi, H. D. U. C. Nguyen, T. H. Nguyen, and N. B. A. O. Nguyen, "Comparative study on traffic accidents and impacts of the enlargement in territory of Hanoi by data analysis," in Proceedings of the Eastern Asia Society for Transportation Studies the 9th International Conference of Eastern Asia Society for Transportation Studies, p. 356, Jeju, Korea, 2011.

[5] T. Q. Vuong, "Traffic conflict technique development for traffic safety evaluation under mixed traffic conditions of developing countries," Journal of Traffic and Transportation Engineering, vol. 5, pp. 228-235, 2017.

[6] R. Li, W. Li, L. Li, C. Zheng, B. Ran, and Y. Cheng, "Crash severity evaluation for unsignalized intersection using conflict 
data," International Journal of Computational Intelligence Systems, vol. 4, no. 6, pp. 1325-1333, 2011.

[7] X.-C. Vuong, R.-F. Mou, and T.-T. Vu, "Safety Impact of timing optimization at mixed-traffic intersections based on simulated conflicts: a case study of Hanoi, Vietnam," in Proceedings of the 2019 4th International Conference on Intelligent Transportation Engineering (ICITE), pp. 247-251, Hanoi, Vietnam, 2019.

[8] L. Zheng, K. Ismail, and X. Meng, "Traffic conflict techniques for road safety analysis: open questions and some insights," Canadian Journal of Civil Engineering, vol. 41, no. 7, pp. 633-641, 2014.

[9] F. H. Amundsen and C. Hyden in Proceedings of the First Workshop on Traffic Conflicts, Oslo, TTI, Oslo, Norway LTH Lund, Sweden, January 1977.

[10] D. Gettman and L. Head, "Surrogate safety measures from traffic simulation models," Transportation Research Record, vol. 1840, no. 1, pp. 104-115, 2003.

[11] C. Hydén, "The development of a method for traffic safety evaluation: the Swedish traffic conflicts technique," Bulletin Lund Institute of Technology Department, vol. 70, 1987.

[12] J. Li, X. Chen, and W. Wang, "Fuzzy evaluation method for the severity of traffic conflicts," Journal of Transportation Systems Engineering and Information Technology, vol. 2, 2008.

[13] X.-P. Hu, A Video-Based Technology to Identify and Determine the Traffic Conflicts at the Intersection, North China University of Technology, China, 2013.

[14] H. Hu, Z. Li, W. Wei, Z. Qu, and Z. Chen, "Fuzzy logic based pedestrian-vehicle conflict discrimination at signalized intersection," Journal of Jilin University (Engineering and Technology Edition), vol. 44, no. 3, 2014.

[15] R. E. N. Gang, G. U. Cheng, L. U. Lili, Z. Zhuping, and D. Chenzi, "Modeling risk degree of conflicts between crossing pedestrians and vehicles at signalized intersections," Journal of Transportation Systems Engineering and Information Technology, vol. 12, no. 5, pp. 76-81, 2012.

[16] J. Yu and L. Wang, "Traffic safety evaluation of urban road intersection based on grey clustering," in Proceedings of the 4th International Conference on Information Technology and Management Innovation, pp. 435-439, Atlantis Press, Amsterdam, Netherlands, October 2015.

[17] M. Huang, J. Zheng, and L. Chi, "Evaluation of traffic safety at intersections in city based on fuzzy clustering," Technology of Economic Areas Communication, vol. 14, no. 4, pp. 84-86, 2012.

[18] T. Sayed and S. Zein, "Traffic conflict standards for intersections," Transportation Planning and Technology, vol. 22, no. 4, pp. 309-323, 1999.

[19] R. Z. A. Majeed and H. A. Ewadh, "A conflict index to assess traffic safety at intersections," In IOP Conference Series: Materials Science and Engineering, vol. 584, no. 1, Article ID 12018, 2019.

[20] R. F. Mou and Q. J. Cai, "Stability evaluation for the surrounding rock structure based on the normal cloud model and the rough set theory," Journal of Safety and Environment, vol. 18, no. 04, pp. 1251-1257, 2018.

[21] H. L. Wang, "The extension and supplement of grey uncertain model," Mathematics in Practice and Theory, vol. 43, no. 16, pp. 231-236, 2013.

[22] L. Deyi, M. Haijun, and S. Xuemei, "Membership clouds and membership cloud generators," Journal of Computer Research and Development, vol. 32, no. 6, pp. 15-20, 1995.

[23] G. Wang, C. Xu, and D. Li, "Generic normal cloud model," Information Sciences, vol. 280, pp. 1-15, 2014.
[24] J.-q. Wang, L. Peng, H.-y. Zhang, and X.-h. Chen, "Method of multi-criteria group decision-making based on cloud aggregation operators with linguistic information," Information Sciences, vol. 274, pp. 177-191, 2014.

[25] K. Di, D. Li, and D. Li, "Cloud theory and its applications in spatial data mining and knowledge discovery," Journal of Image and Graphics, vol. 4, no. 11, pp. 930-935, 1999.

[26] Q. Sun and Y. Wang, "Intelligent sliding mode control of nonlinear systems based on cloud models," in Proceedings of the 29th Chinese Control Conference, pp. 2531-2535, Beijing, China, July 2010.

[27] X.-C. Vuong, R.-F. Mou, H.-S. Nguyen, and T.-T. Vu, "Signal timing optimization of isolated intersection for mixed traffic flow in Hanoi city of Vietnam using VISSIM," in Proceedings of the International Conference on Smart Vehicular Technology, Transportation, Communication and Applications, pp. 133-139, Mount Emei, China, October 2018.

[28] Tracker 6.0.0, https://physlets.org/tracker/ (accessed Jul. 05, 2021), 2021.

[29] D. Li, C. Liu, and W. Gan, "A new cognitive model: cloud model," International Journal of Intelligent Systems, vol. 24, no. 3, pp. 357-375, 2009.

[30] D. Li, D. Cheung, X. Shi, and V. Ng, "Uncertainty reasoning based on cloud models in controllers," Computers \& Mathematics with Applications, vol. 35, no. 3, pp. 99-123, 1998.

[31] J. C. Hayward, Near Miss Determination through Use of a Scale of Danger, 1972.

[32] J. Li, Z. Q. Yue, and S. C. Wong, "Performance evaluation of signalized urban intersections under mixed traffic conditions by gray system theory," Journal of Transportation Engineering, vol. 130, no. 1, pp. 113-121, 2004.

[33] G. R. Brow, "Traffic conflicts for road user safety studies," Canadian Journal of Civil Engineering, vol. 21, no. 1, pp. 1-15, 1994.

[34] J. . H. Kraay, A. R. A. Van Der Horst, and S. Oppe, Manual Conflict Observation Technique DOCTOR, Roadsafety Forall Organization, Voorburg, Netherland, 2013.

[35] World Health Organization, Speed Management: A Road Safety Manual for Decision-Makers and Practitioners, WHO, Geneva, Switzerland, 2008.

[36] J.-L. Martin and D. Wu, "Pedestrian fatality and impact speed squared: c log log modeling from French national data," Traffic Injury Prevention, vol. 19, no. 1, pp. 94-101, 2018.

[37] C. Kong and J. Yang, "Logistic regression analysis of pedestrian casualty risk in passenger vehicle collisions in China," Accident Analysis \& Prevention, vol. 42, no. 4, pp. 987-993, 2010.

[38] F. Cunto, Assessing Safety Performance of Transportation Systems Using Microscopic Simulation, UWSpace, Ontario, Canada, 2008.

[39] V. X. Can, M. Rui-fang, T. Van Hung, and V. T. Thuat, "An analysis of urban traffic incident under mixed traffic conditions based on SUMO: a case study of Hanoi," International Journal of Advanced Research in Engineering and Technology (IJARET), vol. 11, 2020.

[40] Q. V. Tran and A. T. Vu, Analysis of traffic accidents at signalized intersections, Proceedings of the 7th ATRANS Symposium: Young Researcher's Forum 2014-“Transportation for A Better Life: Towards Better ASEAN Connectivity and Safety", Shanghai, China, pp.138-148, 2014.

[41] D. T. M. Hoa, N. H. Duc, N. T. Huong, and N. N. Bao, “On time-distributions of traffic accident and of its related factors: Pilot study with data analysis for an urban district with 
complex traffic situation," In Proceedings of the Eastern Asia Society for Transportation Studies, vol. 9, 2013.

[42] N. Matsuhashi, T. Hyodo, and Y. Takahashi, "Image processing analysis of motorcycle oriented mixed traffic flow in Vietnam," In Proceedings of the Eastern Asia Society for Transportation Studies, vol. 5, pp. 929-944, 2005.

[43] N. Y. Cao and K. Sano, "Estimating capacity and motorcycle equivalent units on urban roads in Hanoi, Vietnam," Journal of Transportation Engineering, vol. 138, no. 6, pp. 776-785, 2012.

[44] T. T. Nguyen, P. T. Cao, and X. C. Vuong, "Analysis traffic conflict technique for traffic safety evaluation at intersection," in Proceedings of the International Conference on Applications of Artificial Intelligence in Transportation (AITC2020), pp. 223-229, Ho Chi Minh City, Vietnam, 2020.

[45] T. L. Saaty, "Decision making with the analytic hierarchy process," International Journal of Services Sciences, vol. 1, no. 1, pp. 83-98, 2008.

[46] X. J. Xie, "Research on material selection with multi-attribute decision method and G1 method," Advanced Materials Research, vol. 952, pp. 20-24, 2014.

[47] J. Liu, Y. Li, Y. Lu, X. Fu, and S. Yan, "Research on the influence factors of ubiquitous power Internet of things for promoting consumption of wind power based on fuzzy G1ISM in China," International Journal of Electrical Power \& Energy Systems, vol. 121, Article ID 106124, 2020.

[48] X. V. Nguyen, Highway Design and Signalized Intersections, Construction Publishing House, Hanoi, Vietnam, 2006. 